\title{
Tumor carcinoide atípico solitario de timo: Una entidad inusual que no debemos olvidar
}

\author{
Natalia Páez J. ${ }^{\star}$, Cristian Valderrama B. ${ }^{3}$, Pedro Chanta M.. , Bernardo Schicht C. \\ 1. Médico radiólogo. Departamento de imágenes diagnósticas. Hospital Eva Perón de San Martín, Buenos Aires, Argentina. \\ 2. Residente de tercer año de radiología. Hospital Eva Perón de San Martín, Buenos Aires, Argentina. \\ 3. Residente de cuarto año de radiología. Hospital Eva Perón de San Martín, Buenos Aires, Argentina. \\ 4. Médico cirujano de Tórax. Departamento de Cirugía de Tórax. Hospital Eva Perón de San Martín, Buenos Aires, Argentina.
}

\section{Solitary atipic carcinoid tumor of the thymus: An unusual entity that we should not forget}

\section{Resumen:}

Los tumores neuroendocrinos primarios (NET) del mediastino son muy raros. Presentamos el caso de un tumor carcinoide atípico primario de timo. Un hombre de 52 años que fue a un examen médico porque se quejaba de tos no productiva sin hemoptisis. Se solicitó una radiografía de tórax donde se evidenció un mediastino notablemente ensanchado, con una tomografía de tórax que mostro una masa tumoral de $90 \times 50 \mathrm{~mm}$ en el mediastino anterosuperior que comprime el arco aórtico y la arteria pulmonar sin un plano de clivaje adecuado. Debido a que el tumor era infiltrativo, se proporcionó escisión quirúrgica completa, quimioterapia y radioterapia al mediastino. El objetivo principal de nuestro estudio fue evaluar los hallazgos de CT y MRI con relación a la literatura mundial. Palabras clave: Timo; Tumor carcinoide; Tumor neuroendocrino.

\begin{abstract}
:
Primary neuroendocrine tumors (NET) of the mediastinum are very rare. We present the case of a primary atypical carcinoid tumor of the thymus. A 52-year-old man who went to a medical examination because he complained of a non-productive cough without hemoptysis. A chest radiograph was requested where a markedly widened mediastinum was evidenced, with a thoracic tomography showing a tumor mass of 90 $x 50 \mathrm{~mm}$ in the anterosuperior mediastinum that compresses the aortic arch and pulmonary artery without an adequate cleavage plane. Because the tumor was infiltrative, complete surgical excision, chemotherapy and radiotherapy were provided to the mediastinum. The main objective of our study was to evaluate the findings of $C T$ and $M R I$ in relation to universal literature.
\end{abstract}

Keywords: Carcinoid tumor; Neuroendocrine tumor; Thymus.

Páez N., et al. Tumor carcinoide atípico solitario de timo: Una entidad inusual que no debemos olvidar. Rev Chil Radiol 2020; 26(3): 120-124.

*Correo electrónico: Natalia Páez Jaramillo / natypaez1987@gmail.com.

Trabajo enviado el 11 de enero de 2020. Aceptado para publicación el 03 de julio de 2020.

\section{Introducción}

El tumor neuroendocrino primario de timo constituye una enfermedad muy poco frecuente que se confunde con otro tipo de tumores de origen timico. Los tumores neuroendocrinos del timo, según la clasificación de la Organización Mundial de la Salud (OMS) del año 2015, se incluyen dentro de los tumores epiteliales malignos.

En esta última clasificación se nombran aparte de los demás tumores epiteliales, subclasificandolos como tumor carcinoide tímico y carcinoide atípico'. Con frecuencia se asocian a niveles elevados de corticotropina (ACTH) en la sangre. En el tejido tumoral se detectan una serie de marcadores neuroendocrinos entre los que ocupan un papel destacado la cromogranina, la citoqueratina, la sinaptofisina y la enolasa neuroespecífica ${ }^{3}$. 


\section{Presentación del caso}

Paciente masculino de 52 años, con antecedentes de hipertensión arterial con episodios de tos con expectoración mucoide esporádica sin hemoptisis y respuesta parcial terapéutica, presentando exacerbación de la sintomatología en el último año por lo cual consulta.

Hallazgos positivos al examen físico: Ganglios supraclaviculares izquierdos palpables menores a $1 \mathrm{~cm}$.

\section{Hallazgos imagenológicos}

Se realizaron los siguientes estudios de imagen (Figuras 1, 2 y 3 ).
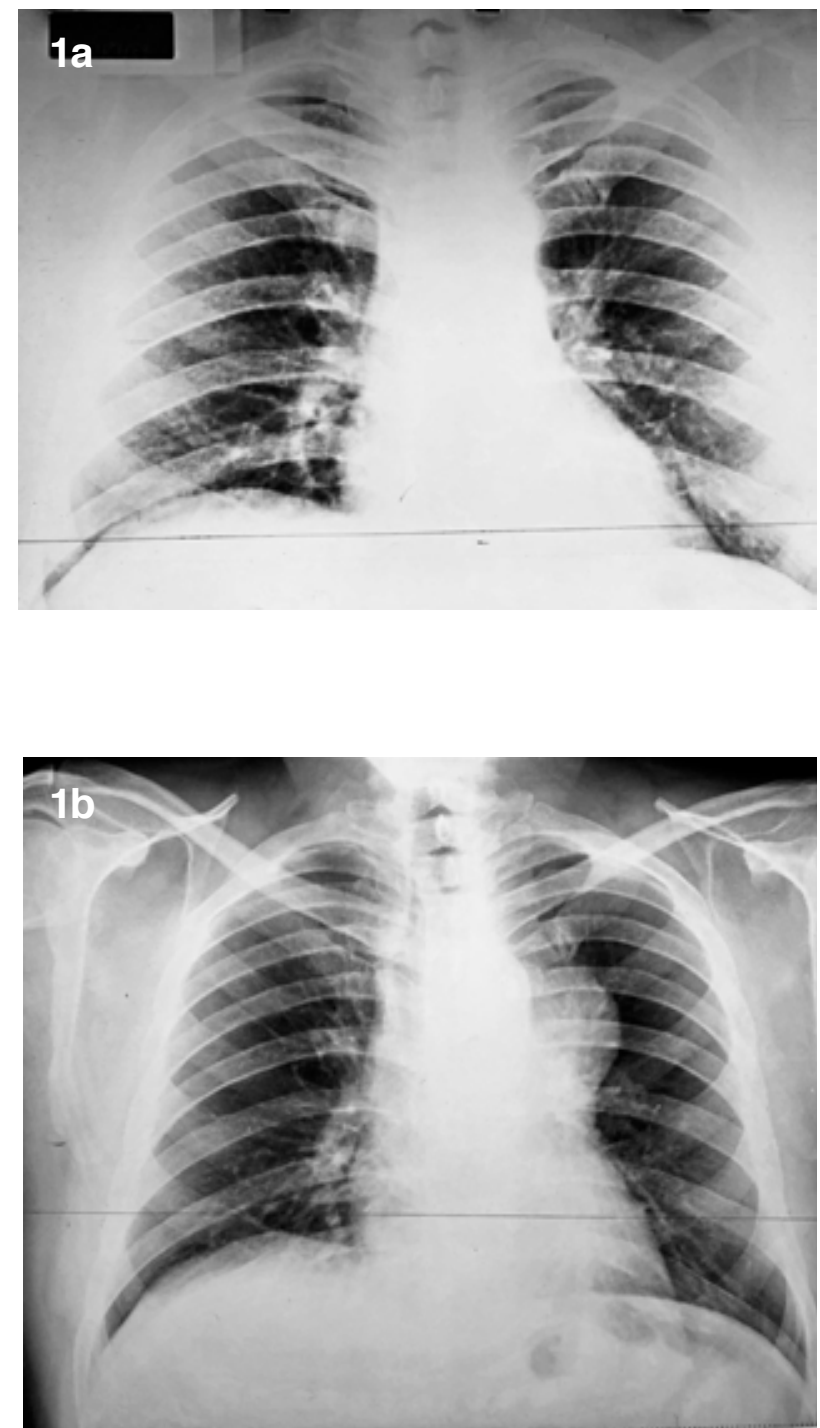

Figuras 1a y b: Radiografía de tórax (PA). a). Radiografía de tórax de octubre 2017: Normal. b). Radiografía de tórax de noviembre 2018: Imagen radiodensa en región parahiliar izquierda de bordes bien delimitados.
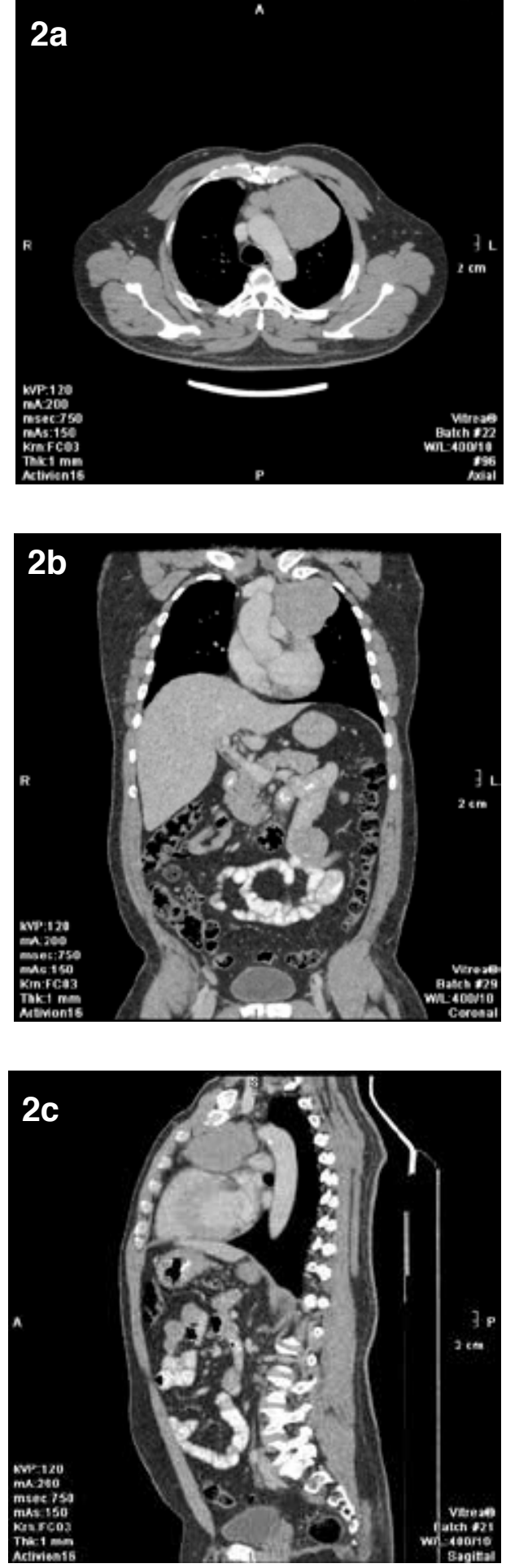

Figuras 2a, $\boldsymbol{b}$ y $\boldsymbol{c}:$ TC de tórax con contraste endovenoso (a: corte axial, b: corte coronal y c: corte sagital). Formación con densidad de partes blandas de bordes bien definidos $(90 \times 50 \mathrm{~mm})$ a nivel de mediastino anterior y región prevascular que comprime el cayado aórtico y el tronco de la arteria pulmonar con captación discretamente heterogénea tras la administración de contraste endovenoso (corte axial, coronal y sagital). Presenta también 3 imágenes de similares características de menor tamaño en mediastino anterior. No se registro derrame pleural ni pericárdico. 

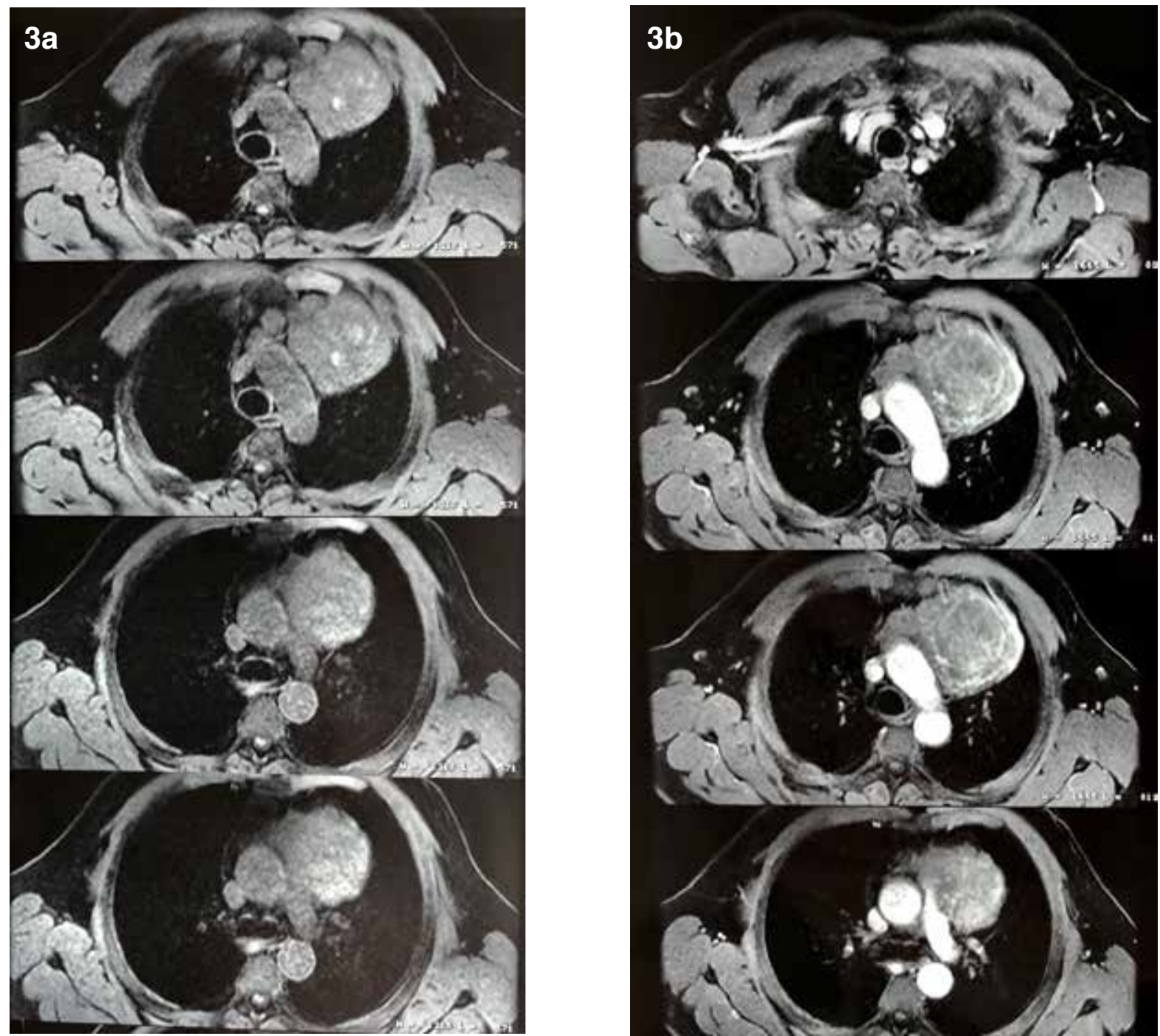

Figuras 3a y b: Angioresonancia de tórax, cortes axiales en secuencia T1 sin y con gadolinio. Formación de densidad de partes blandas en mediastino superior en situación antero lateral izquierda al cayado aórtico, lobulada con señal levemente heterogénea y pequeñas áreas hiperintensas (por probable microsangrados) en secuencia T1 sin contraste. El realce es intenso y de forma irregular luego de la administración de gadolinio, sugiriendo un proceso neoproliferativo.

\section{Estudios complementarios}

Se solicitaron niveles del ácido 5-hidroxiindolacético en orina de $24 \mathrm{~h}$ con resultado de $1.8 \mathrm{mg} / 24$ horas (negativo) descartando tumores carcinoides metastasicos funcionantes.

Se realizó perfil bioquímico incluyendo perfil hepático, renal y lipídico para descartar otras entidades asociadas con la neoplasia neuroendocrina múltiple tipo 1 o NEM 1 (afección pancreática, paratiroidea e hipofisaria), los cuales fueron negativos.

Posteriormente fue llevado a una biopsia percutánea por trucut con resultado que mostro histología e inmunohistoquímica compatible con tumor neuroendocrino (tumor carcinoide atípico).

\section{Conducta terapéutica}

Las evaluaciones funcionales respiratorias y cardiológica revelaron parámetros dentro de los limites de la normalidad, por lo que se decidió la intervención quirúrgica previa neoadyuvancia con 3 ciclos de quimioterapia (ectoposido y CDDP) con poca reducción del tamaño tumoral. Se realizó reseccion del tumor, el cual presentaba un diámetro aproximado de $10 \times 10 \mathrm{x}$ $10 \mathrm{~cm}$ y 3 lesiones nodulares de similares características adyacentes, explorándose pericardio y grandes vasos, con evidencia de infiltración del pericardio. Tanto la arteria pulmonar como la vena innominada, presentaban signos macroscópicos de infiltración por dichas lesiones dado que se encontraban en íntimo 
contacto con el pericardio infiltrado. Se considera resección tumoral aproximada del 98\%. La lesión resecada fue llevada a estudio anatomopatológico confirmando el diagnóstico.

Posteriormente el paciente presenta mejoría de su condición clínica, sin recidiva de la enfermedad en los controles imagenológicos (Figura 4). Se encuentra actualmente en sesiones de radioterapia, en seguimiento interdisciplinario, presentando como complicación post radioterapia disfonía y parálisis frénica por lo cual continua con terapia física y respiratoria.
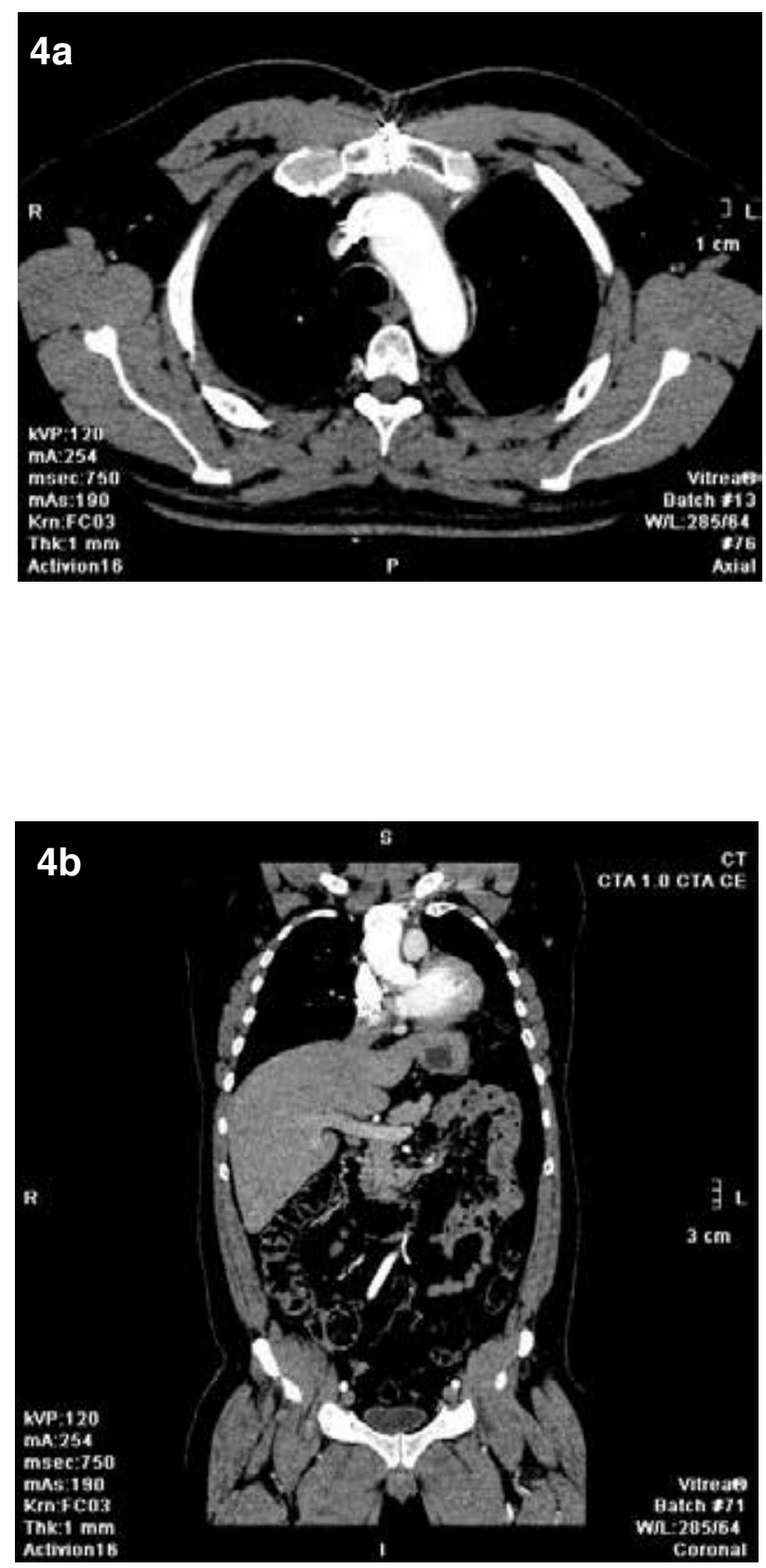

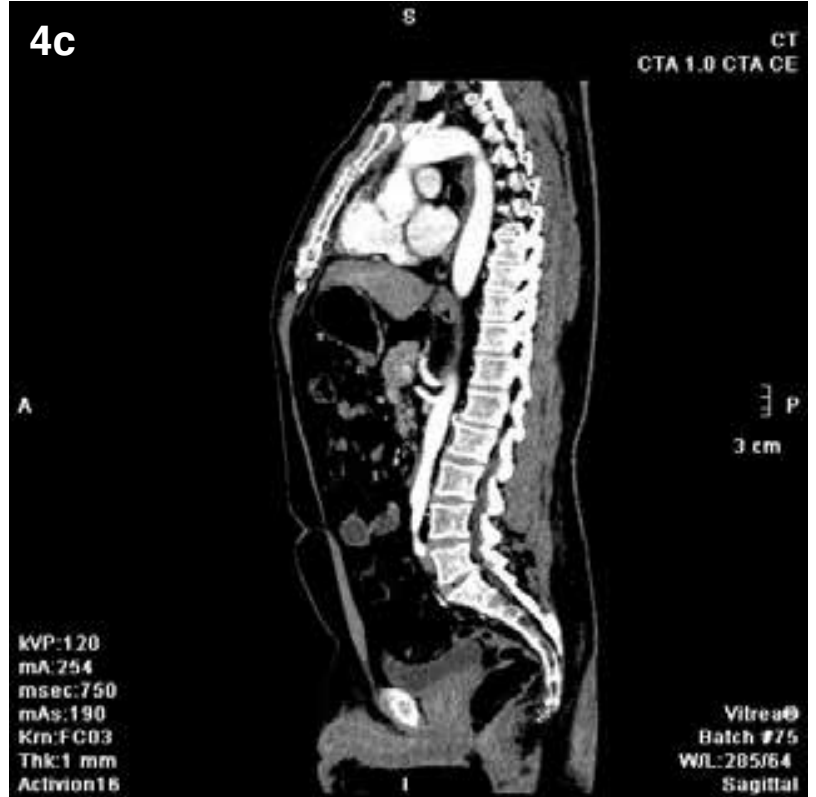

Figura 4: (a) corte axial, (b) corte coronal y (c) corte sagital). TC de tórax, abdomen y pelvis con contraste endovenoso (25/09/2019). Control posquirúrgico sin evidencia de recidiva locorregional de la enfermedad ni compromiso metastásico a otros órganos.

\section{Discusión}

Los tumores neuroendocrinos tímicos son tumores epiteliales de la glándula del timo que están compuestos predominante o exclusivamente por células neuroendocrinas, que se pueden demostrar mediante tinciones en inmunohistoquímica ${ }^{6}$.

Los tumores neuroendocrinos tímicos son poco frecuentes y constituyen del 2 al $5 \%$ de todos los tumores epiteliales tímicos. Según la Organización Mundial de la Salud, se clasifican en 2 categorías principales: bien diferenciados que comprenden el carcinoide típico y atípico, y pobremente diferenciados que comprenden el carcinoma neuroendocrino de células grandes y de células pequeñas ${ }^{6,7}$.

Aproximadamente el $50 \%$ de los pacientes con carcinoide tímico tienen anormalidades endocrinas, más comúnmente el Síndrome de Cushing, debido a la producción ectópica de ACTH o están asociados a un síndrome de neoplasia endocrina múltiple (MEN por sus siglas en inglés), específicamente MEN-Tipo $1^{8}$.

Los carcinoides atípicos son principalmente tumores de adultos y existe predominio en hombres ${ }^{7}$. Suelen presentar signos y síntomas inespecíficos, si bien aproximadamente el 50\% carecen de ma- 
nifestaciones clínicas evidentes ${ }^{5}$ y un tercio de los pacientes se encuentran asintomáticos, por lo que las particularidades clínicas detalladas de estos tumores no están bien definidas.

Algunos pacientes con carcinoides tímicos ocultos o tempranos tienen hallazgos normales en las radiografías de tórax. Estos individuos pueden presentar signos clínicos de producción ectópica de ACTH. La tomografía computarizada, la resonancia magnética y la medicina nuclear pueden ser útiles para evaluar a estos pacientes (con lesiones ocultas y hormonalmente activas) ${ }^{7}$.

El timoma o carcinoma tímico es una enfermedad importante que debe incluirse como diagnóstico diferencial del carcinoide tímico. El gran tamaño del tumor y los focos necróticos son características comunes compartidas entre los carcinoides y los timomas invasivos ${ }^{8}$.

Rosado de Christenson et.al., informaron la presencia de márgenes agudos, contornos lobulados, invasión mediastínica y metástasis a distancia en las imágenes de CT como una característica del carcinoide tímico ${ }^{7}$.

En nuestro caso de acuerdo con lo revisado en la literatura mundial, encontramos que tanto la edad, el género y el cuadro clínico de presentación coincidieron con lo reportado en otras revisiones. En relación con los hallazgos imagenológicos de resonancia encontramos que la lesión en mediastino anterior descrita presentaba señal levemente heterogénea y pequeñas áreas hiperintensas (por probable microsangrados) en secuencia $\mathrm{T} 1$ sin contraste. Tras la administración de gadolinio presenta realce intenso y de forma irregular en secuencia $\mathrm{T}^{2,4}$.

\section{Referencias}

1. TravisD., et al. The 2015 World Health Organization Classification of Lung Tumors. Impact of Genetic, Clinical and Radiologic Advances Since the 2004 Classification. J Thorac Oncol. 2015 Sep; 10(9): 1243-1260.

2. Shimamoto A, Ashizawa K, Kido Y, Hayashi H, Nagayasu T, Kawakami A, et al. CT and MRI findings of thymic carcinoid. Br J Radiol 2017; 90: 20150341.

3. Marquez-Medina D, Popat S. Systemic therapy for pulmonary carcinoids. Lung Cancer 2015; 90(2): 139147.

4. Dutta R, Kumar A, Julka PK, Mathur SR, Kaushal S, Kumar R, et al. Thymic neuroendocrine tumour (carcinoid): clinicopathological features of four patients with different presentation. Interact Cardiovasc Thorac Surg 2010; 11: 732-736. doi: https://doi.org/10.1510/ icvts.2010.237651.

5. Marx A, Shimosato Y, Kuo TT, Chan JK, Travis WD, Wick WR. Tumours of the thymus: thymic neuroendocrine tumours. In: Travis WD, Brambilla E, Müller-Hermelink HK, Harris CC. World Health Organization classification of tumours. Pathology and genetics of tumours of the lung, pleura, thymus and heart. Lyon, France: IARC Press. 2004; 188-197.

6. Strollo DC, Rosado de Christenson ML, Jett JR. Primary mediastinal tumors. Part 1: tumors of the anterior mediastinum. Chest 1997; 112: 511-522. doi: https://doi. org/10.1378/chest.112.2.511.

7. Rosado de Christenson ML, Abbott GF, Kirejczyk WM, Galvin JR, Travis WD. Thoracic carcinoids: radiologic-pathologic correlation. Radiographics 1999; 19: 707-736. doi: https://doi.org/10.1148/ radiographics.19.3.g99ma11707.

8. Restrepo CS, Pandit M, Rojas IC, Villamil MA, Gordillo $\mathrm{H}$, Lemos $\mathrm{D}$, et al. Imaging findings of expansile lesions of the thymus. Curr Probl Diagn Radiol. 2005; 34: 2234. doi: https://doi.org/10.1067/j.cpradiol.2004.10.001. 